\title{
System Size Resonance in Coupled Noisy Systems and in the Ising Model
}

\author{
A. Pikovsky and A. Zaikin \\ Department of Physics, University of Potsdam, Postfach 601553, D-14415 Potsdam, Germany \\ M. A. de la Casa \\ Departamento Física Fundamental, Universidad Nacional de Educación a Distancia, 28040 Madrid, Spain
}

(Received 8 March 2001; published 16 January 2002)

\begin{abstract}
We consider an ensemble of coupled nonlinear noisy oscillators demonstrating in the thermodynamic limit an Ising-type transition. In the ordered phase and for finite ensembles stochastic flips of the mean field are observed with the rate depending on the ensemble size. When a small periodic force acts on the ensemble, the linear response of the system has a maximum at a certain system size, similar to the stochastic resonance phenomenon. We demonstrate this effect of system size resonance for different types of noisy oscillators and for different ensembles - lattices with nearest neighbors coupling and globally coupled populations. The Ising model is also shown to demonstrate the system size resonance.
\end{abstract}

DOI: 10.1103/PhysRevLett.88.050601

Stochastic resonance has attracted much interest recently [1]. As was demonstrated in [2], a response of a noisy nonlinear system to a periodic forcing can exhibit a resonancelike dependence on the noise intensity. In other words, there exists a "resonant" noise intensity at which the response to a periodic force is maximally ordered. Stochastic resonance has been observed in numerous experiments [3]. Noteworthy, the order in a noise-driven system can have a maximum at a certain noise level even in the absence of periodic forcing, this phenomenon being called coherence resonance [4].

Being first discussed in the context of a simple bistable model, stochastic resonance has been also studied in complex systems consisting of many elementary bistable cells [5]; moreover, the resonance may be enhanced due to coupling [6]. In this paper we discuss another type of resonance in such systems, namely, the system size resonance, when the dynamics is maximally ordered at a certain number of interacting subsystems. Contrary to previous reports of array-enhanced stochastic resonance (cf. also [7]), here we fix the noise strength, coupling, and other parameters; only the size of the ensemble changes.

The basic model to be considered below is the ensemble of noise-driven bistable overdamped oscillators, governed by the Langevin equations,

$$
\dot{x}_{i}=x_{i}-x_{i}^{3}+\frac{\varepsilon}{N} \sum_{j=1}^{N}\left(x_{j}-x_{i}\right)+\sqrt{2 D} \xi_{i}(t)+f(t) \text {. }
$$

Here $\xi_{i}(t)$ is a Gaussian white noise with zero mean: $\left\langle\xi_{i}(t) \xi_{j}\left(t^{\prime}\right)\right\rangle=\delta_{i j} \delta\left(t-t^{\prime}\right) ; \varepsilon$ is the coupling constant; $N$ is the number of elements in the ensemble, and $f(t)$ is a periodic force to be specified later. In the absence of periodic force, the model (1) has been extensively studied in the thermodynamic limit $N \rightarrow \infty$. It demonstrates an Isingtype phase transition at $\varepsilon=\varepsilon_{c}$ from the disordered state with vanishing mean field $X=N^{-1} \sum_{i} x_{i}$ to the "ferromagnetic" state with a nonzero mean field $X= \pm X_{0}$ [8].
PACS numbers: 05.40.Ca, 05.45. $-\mathrm{a}, 05.50 .+\mathrm{q}$

While in the thermodynamic limit the full description of the dynamics is possible, for finite system sizes we have mainly a qualitative picture: In the ordered phase the mean field $X$ switches between the values $\pm X_{0}$ and its average vanishes for all couplings. The rate of switchings depends on the system size and tends to zero as $N \rightarrow \infty$.

For us, the main importance is the fact that qualitatively the behavior of the mean field can be represented as the noise-induced dynamics in a potential with one minimum in the disordered phase (at $X=0$ ) and two symmetric minima (at $X= \pm X_{0}$ ) in the ordered phase. Now, applying the ideas of the stochastic resonance, one can expect in the bistable case (i.e., in the ordered phase for small enough noise or for large enough coupling) a resonantlike behavior of the response to a periodic external force when the intensity of the effective noise is changed. Because this intensity is inverse proportional to $N$, we obtain the resonancelike curve of the response in dependence of the system size. The main idea behind the system size resonance is that in finite ensembles of noise-driven or chaotic systems the dynamics of the mean field can be represented as driven by the effective noise whose variance is inverse proportional to the system size [9]. This idea has been applied to the description of a transition to collective behavior in [10]. In [11] it was demonstrated that the finite-size fluctuations can cause a transition that disappears in the thermodynamic limit.

Before proceeding to a quantitative analytic description of the phenomenon, we illustrate it with direct numerical simulations of the model (1), with a forcing term $f(t)=$ $A \cos (\Omega t)$. Figure 1 shows the linear response function, i.e., the ratio of the spectral component in the mean field at frequency $\Omega$ and the amplitude of forcing $A$, in the limit $A \rightarrow 0$. For a given frequency $\Omega$ the dependence on the system size is a bell-shaped curve, with a pronounced maximum. The dynamics of the mean field $X(t)$ is illustrated in Fig. 2, for three different system sizes. The resonant dynamics (Fig. 2b) demonstrates a typical for 


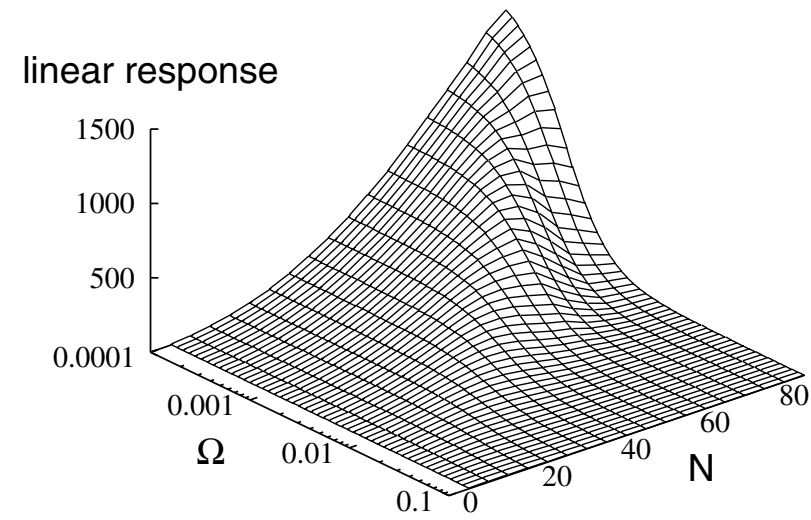

FIG. 1. Linear response of the ensemble (1) $(D=0.5, \varepsilon=2)$ in dependence on the frequency and the system size $N$.

stochastic resonance synchrony between the driving periodic force and the switchings of the field between the two stable positions.

To describe the system size resonance analytically, we use, following [8], the Gaussian approximation. In this approximation, one writes $x_{i}=X+\delta_{i}$ and assumes that $\delta_{i}$ are independent Gaussian random variables with zero mean and the variance $M$. Assuming furthermore that $N^{-1} \sum_{i} \delta_{i}^{2}=M$ and neglecting the odd moments $N^{-1} \sum_{i} \delta_{i}, N^{-1} \sum_{i} \delta_{i}^{3}$, as well as the correlations between $\delta_{i}$ and $\delta_{j}$, we obtain from (1) the equations for $X$ and $M$ :

$$
\begin{gathered}
\dot{X}=X-X^{3}-3 M X+\sqrt{\frac{2 D}{N}} \eta(t)+f(t), \\
\frac{1}{2} \dot{M}=M-3 X^{2} M-3 M^{2}-\varepsilon M+D,
\end{gathered}
$$

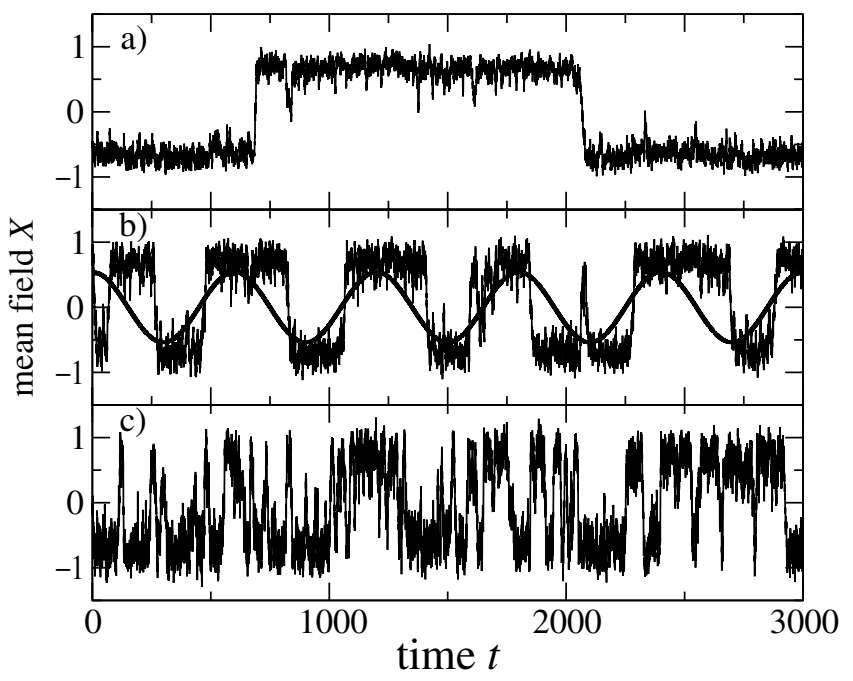

FIG. 2. The time dependence of the mean field in the ensemble (1) for $D=0.5, \varepsilon=2, A=0.02, \Omega=\pi / 300$, and different sizes of the ensemble: (a) $N=80$, (b) $N=35$, and (c) $N=$ 15. We also depict the periodic force (its amplitude is not in scale) to demonstrate the synchrony of the switchings with the forcing in (b). where $\eta$ is the Gaussian white noise having the same properties as $\xi_{i}(t)$. In the thermodynamic limit $N \rightarrow \infty$ the noisy term $\eta$ vanishes. If the forcing term is absent $(f=0)$, the equations coincide with those derived in [8]. This system of coupled nonlinear equations exhibits a pitchfork bifurcation of the equilibrium $X=0, M>0$ at $\varepsilon_{c}=3 D$. This bifurcation is supercritical for $D>2 / 3$ in accordance with the exact solution of (1) given in [8]; below we consider only this case. For $\varepsilon>\varepsilon_{c}$ the system is bistable with two symmetric stable fixed points,

$$
X_{0}^{2}=(2-\varepsilon+S) / 4, \quad M_{0}=(2+\varepsilon-S) / 12
$$

[here $S=\sqrt{(2+\varepsilon)^{2}-24 D}$ ], and the unstable point $X=0, M=\left[1-\varepsilon+\sqrt{(1-\varepsilon)^{2}+12 D}\right] / 6$. Now, with the external noise $\eta$ and with the periodic force $f(t)$, the problem reduces to a standard problem in the theory of stochastic resonance, i.e., to the problem of the response of a noise-driven nonlinear bistable system to an external periodic force (because the noise affects only the variable $X$, it does not lead to unphysical negative values of variance $M$, since $\dot{M}$ is strictly positive at $M=0$ ).

To obtain an analytical formula, we perform further simplification of the system (2) and (3). Near the bifurcation point, we can use the slaving principle to obtain a standard noise-driven bistable system:

$$
\dot{X}=a X-b X^{3}+\sqrt{\frac{2 D}{N}} \eta(t)+f(t),
$$

where $\quad a=1+0.5(\varepsilon-1)-0.5 \sqrt{(\varepsilon-1)^{2}+12 D}$, $b=-0.5+1.5(\varepsilon-1)\left[(\varepsilon-1)^{2}+12 D\right]^{-1 / 2}$. A better approximation valid also beyond a vicinity of the critical point can be constructed if we use $\bar{b}=a X_{0}^{-2}$ instead of $b$, where the fixed point $X_{0}$ is given by (4). Having written the ensemble dynamics as a standard noise-driven double-well system (5) (cf. [1,12]), we can use the analytic formula for the linear response $R$ derived in [12]. It reads

$$
R=\frac{N X_{0}^{2}}{2 D a}\left(\frac{\mathcal{D}_{-3 / 2}(-\sqrt{s})}{\mathcal{D}_{-1 / 2}(-\sqrt{s})}\right)^{2}\left[1+\frac{\pi^{2} \Omega^{2}}{2 a^{2}} \exp (s)\right]^{-1},
$$

where $s=a N X_{0}^{2} /(2 D)$, and $\mathcal{D}$ are the parabolic cylinder functions. We compare the theoretical linear response function with the numerically obtained one in Fig. 3. The qualitative correspondence is good; moreover, the maxima of the curves are rather good reproduced with the formula (6).

Above, we concentrated on the properties of the linear response. Numerical simulations with the finite forcing amplitude yielded the results similar to that presented in Figs. 1 and 3. However, for large amplitudes of forcing (e.g., $A>0.1$ for $\Omega=0.01, D=0.5, \varepsilon=2$ ) a saturation was observed: Here the response grows monotonically with $N$. This is in full agreement with the corresponding property of the stochastic resonance in double-well systems of type (5), where the saturation 


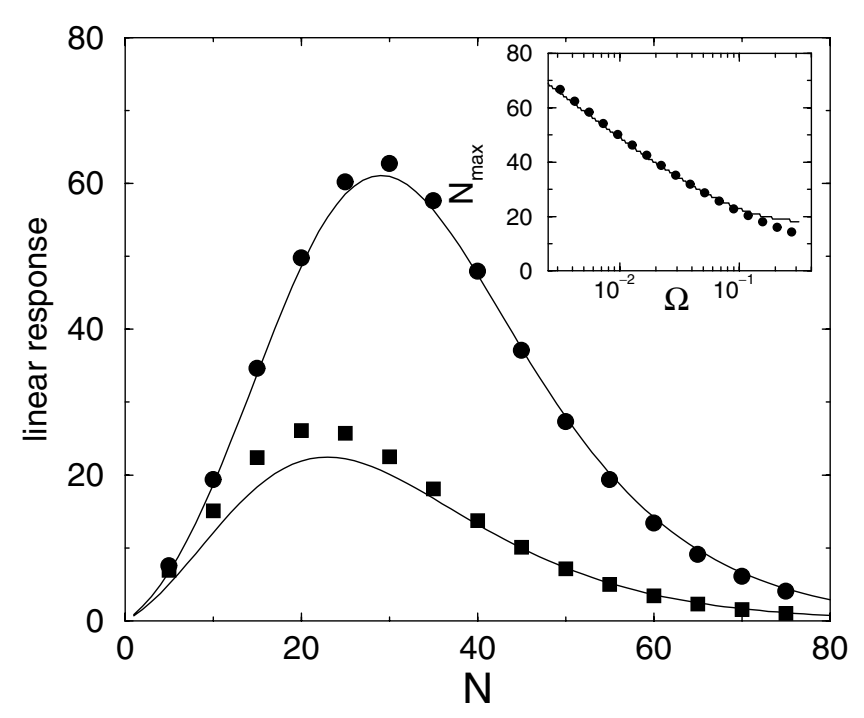

FIG. 3. Comparison of the system size dependencies of the linear response function for frequencies $\Omega=0.05$ (circles) and $\Omega=0.1$ (squares) with theory (6). The parameters are $D=1$ and $\varepsilon-\varepsilon_{c}=2.5$ (where the exact $\varepsilon_{c}$ and the approximate $\varepsilon_{c}=3 D$ are used for the ensemble and the Gaussian approximation, respectively). Inset: Dependence of the system size yielding maximal linear response on the driving frequency $\Omega$ [circles: simulations of the ensemble (1), line is obtained by maximizing the expression (6)].

occurs for small noise intensities (cf. Fig. 7 in [1]), due to the disappearance of multistability for large forcing amplitudes.

It is instructive to compare the response of the noisedriven system (1) with the noise-free case $D=0$. Without external force, the ensemble relaxes eventually to a steady state solution with some mean field $X$; in this state each oscillator can be in one of the stable steady positions of the potential; correspondingly, the oscillators form one or two clusters. From the clustering it follows that the linear response does not depend on the number of elements in the ensemble. Our numerical experiments demonstrated also that the response is system size independent for large forcing amplitudes as well, where, e.g., the force-induced cluster mergings occur. Thus, the effect of system size resonance essentially relies on the presence of noise, which breaks the clustering.

Above, we have considered the system of globally coupled nonlinear oscillators (1). The same effect of system size resonance can be observed in a lattice with nearest neighbors coupling as well. In the thermodynamic limit, the Ising-type phase transition occurs in the lattice (if its dimension is larger than one). Similar to the globally coupled ensemble, in finite lattices in the ordered phase the switchings between the two stable states of the mean field are observed. With the same argumentation as above, we can conclude that the response of the mean field to a periodic forcing can have a maximum at a certain lattice size, while all other parameters (noise intensity, cou- pling strength, etc.) are kept constant. We illustrate this in Fig. 4.

As the next example we consider the two-dimensional nearest neighbor Ising model in the presence of a timedependent external field. The Hamiltonian of the system reads

$$
H=-J \sum_{\langle i j\rangle} s_{i} s_{j}-A \cos (\Omega t) \sum_{i} s_{i},
$$

where $J>0$ and $s_{i}= \pm 1$. We are interested in the dependence of the response of the mean magnetization $m(t)=$ $\frac{1}{N} \sum_{i} s_{i}(t)$ on the system size $N$ (for the usual stochastic resonance in the Ising model, i.e., for the dependence of the response on the temperature, see [13]). To calculate the linear response, we used the fluctuation-dissipation theorem and obtained this quantity by virtue of the power spectrum of fluctuations of $m(t)$. The latter was found using the Metropolis Monte Carlo method on a lattice with helical boundary conditions [14]. The results presented in Fig. 5 demonstrate the system size resonance of the linear response in the two-dimensional Ising model.

As the last example of the system size resonance, we consider a lattice where each individual element does not exhibit bistable noisy dynamics, but such a behavior appears due to interaction and multiplicative noise. This model is described by the set of Langevin equations $[15,16]$ :

$$
\begin{aligned}
\dot{x}_{i}= & -x_{i}\left(1+x_{i}^{2}\right)^{2}+\frac{\varepsilon}{K} \sum_{j}\left(x_{j}-x_{i}\right) \\
& +\sqrt{2 D} \xi_{i}(t)\left(1+x_{i}^{2}\right)+f(t) .
\end{aligned}
$$

As has been demonstrated in [15], in some region of couplings the $\varepsilon$ system (8) exhibits the Ising-type transition.

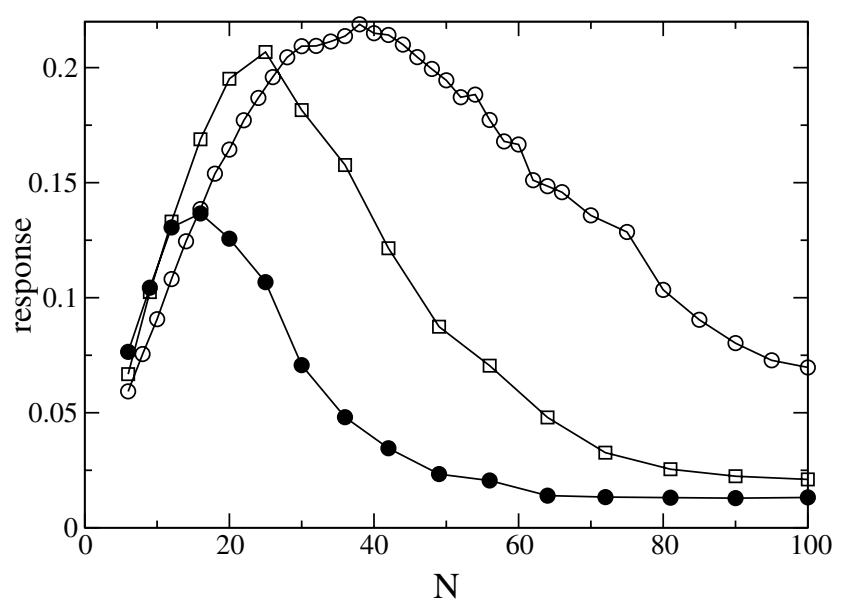

FIG. 4. Filled circles: Response of a two-dimensional lattice of $N$ with nearest neighbors coupling for $A=0.02, T=500$, $D=0.5$, and $\varepsilon=4$. Squares: Response of system (8) (a twodimensional lattice with $D=1.25, \varepsilon=30, A=0.1$, and $T=$ 140). Circles: The same as squares, but for a globally coupled lattice with $D=1, \varepsilon=20, A=0.1$, and $T=100$. 


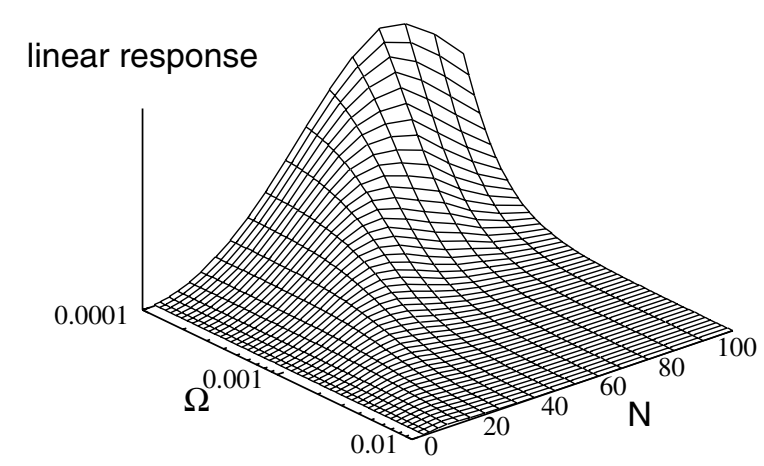

FIG. 5. Linear response (in arbitrary units) of the Ising model (7) for the temperature $T=2 J$ slightly below the critical temperature $T_{c}=2.269 \mathrm{~J}$.

If an additional additive noise is added to (8), then one observes transitions between these states and the so-called double stochastic resonance in the presence of the periodic forcing [17]. As is evident from the considerations above, such transitions occur even in the absence of the additive noise if the system is finite. Thus, the system size resonance should be observed in the lattice (8) as well. We confirm this in Fig. 4.

Another possible field of application of the system size resonance is the neuronal dynamics (see, e.g., [18]). Individual neurons have been demonstrated to exhibit stochastic resonance $[3,19]$. While in experiments one can easily adjust noise to achieve the maximal sensitivity to an external signal, it may not be obvious how this adjustment takes place in nature. The above consideration shows that changing the number of elements in a small ensemble of coupled bistable elements to the optimum can significantly improve the sensitivity (cf. [5]). Moreover, changing its connectivity and/or coupling strength, a neuronal system can tune itself to signals with different frequencies.

In conclusion, we have shown that, in populations of coupled noise-driven elements, exhibiting in the thermodynamic limit the Ising-type transition, in the ordered phase (i.e., for relatively small noise and large coupling) the response to a periodic force achieves maximum at a certain size of the system. We demonstrated this effect for the Ising model, as well as for lattices and globally coupled ensembles of noisy oscillators. We expect the system size resonance to occur also in purely deterministic systems demonstrating the Ising-type transition, e.g., in the MillerHuse coupled map lattice [20]. The system size resonance is described theoretically by reducing the dynamics of the mean field to a low-dimensional bistable model with an effective noise that is inverse proportional to the system size. The stochastic resonance in the mean field dynamics then manifests itself as the system size resonance.

We thank N. Brilliantov, A. Neiman, J. Parrondo, F. J. de la Rubia, and R. Toral for useful discussions, and M. Rosenblum for help in analysis of Eq. (6). M.C. thanks the DGESEIC Project No. PB97-0076 and the
DGES Grantship No. AP98 07249358. A.Z. acknowledges financial support from ESA.

[1] L. Gammaitoni, P. Hänggi, P. Jung, and F. Marchesoni, Rev. Mod. Phys. 70, 223 (1998); P. Jung, Phys. Rep. 234, 175 (1993).

[2] R. Benzi, A. Sutera, and A. Vulpiani, J. Phys. A 14, L453 (1981).

[3] A. Longtin, A. Bulsara, and F. Moss, Phys. Rev. Lett. 67, 656 (1991); A. Simon and A. Libchaber, Phys. Rev. Lett. 68, 3375 (1992); M.L. Spano, M. Wun-Folge, and W. L. Ditto, Phys. Rev. A 46, 5253 (1992); S. Barbay, G. Giacomelli, and F. Marin, Phys. Rev. E 61, 157 (2000).

[4] A. Pikovsky and J. Kurths, Phys. Rev. Lett. 78, 775 (1997); A. Neiman, P. I. Saparin, and L. Stone, Phys. Rev. E 56, 270 (1997).

[5] P. Jung, U. Behn, E. Pantazelou, and F. Moss, Phys. Rev. A 46, R1709 (1992); M. Morillo, J. Gómez-Ordoñez, and J.M. Casado, Phys. Rev. E 52, 316 (1995); J. M. Casado and M. Morillo, Phys. Rev. E 52, 2088 (1995); H. Gang, H. Haken, and X. Fagen, Phys. Rev. Lett. 77, 1925 (1996); P. Jung and G. Mayer-Kress, Phys. Rev. Lett. 74, 2130 (1995).

[6] J. F. Lindner et al., Phys. Rev. Lett. 75, 3 (1995); Phys. Rev. E 53, 2081 (1996).

[7] A. Neiman, L. Schimansky-Geier, and F. Moss, Phys. Rev. E 56, R9 (1997); B. Hu and C. Zhou, Phys. Rev. E 61, R1001 (2000).

[8] R. C. Desai and R. Zwanzig, J. Stat. Phys. 19, 1 (1978).

[9] D. A. Dawson and J. Gärtner, Stochastics 20, 247 (1987); A. S. Pikovsky and J. Kurths, Physica (Amsterdam) 76D, 411 (1994); A. Hamm, Physica (Amsterdam) 142D, 41 (2000).

[10] A. Pikovsky and S. Ruffo, Phys. Rev. E 59, 1633 (1999).

[11] A. S. Pikovsky, K. Rateitschak, and J. Kurths, Z. Phys. B 95, 541 (1994).

[12] P. Jung and P. Hänggi, Phys. Rev. A 44, 8032 (1991).

[13] Z. Néda, Phys. Rev. E 51, 5315 (1995); J. Javier Brey and A. Prados, Phys. Lett. A 216, 240 (1996); U. Siewert and L. Schimansky-Geier, Phys. Rev. E 58, 2843 (1998).

[14] M. E. J. Newmann and G. T. Barkema, Monte Carlo Methods in Statistical Physics (Clarendon, Oxford, 1999).

[15] C. V. der Broeck, J. M. R. Parrondo, and R. Toral, Phys. Rev. Lett. 73, 3395 (1994); C. V. der Broeck et al., Phys. Rev. E 55, 4084 (1997).

[16] P. Landa, A. Zaikin, and L. Schimansky-Geier, Chaos Solitons Fractals 9, 1367 (1998).

[17] A. A. Zaikin, J. Kurths, and L. Schimansky-Geier, Phys. Rev. Lett. 85, 227 (2000); A. A. Zaikin, K. Murali, and J. Kurths, Phys. Rev. E 63, 020103(R) (2001).

[18] P. A. Tass, Phase Resetting in Medicine and Biology: Stochastic Modelling and Data Analysis (Springer-Verlag, Berlin, 1999).

[19] J. K. Douglas, L. Wilkens, E. Pantazelou, and F. Moss, Nature (London) 365, 337 (1993); D. F. Russell, L. A. Wilkens, and F. Moss, Nature (London) 402, 291 (1999).

[20] J. Miller and D. A. Huse, Phys. Rev. E 48, 2528 (1993). 